\title{
Différences culturelles et relations d'affaires entre pays d'Afrique et pays émergents $\mathrm{d}^{\prime} \mathrm{Asie}^{1}$
}

La question de I'influence des valeurs culturelles dans les relations d'affaires entre I'Afrique et I'Asie a rarement fait l'objet d'attention dans les travaux consacrés aux études comparatives en matière de management. Cette contribution vise à mettre en évidence les différences et convergences culturelles fondamentales susceptibles d'influencer les négociations commerciales entre I'Afrique et I'Asie. Ainsi sur la base de trois enquêtes cross-culturelles et l'usage de la notion de distance culturelle, il est établi que I'orientation à long terme des pays asiatiques, reflet de la pensée confucéenne, contraste fortement avec les valeurs caractéristiques de « la sagesse africaine ». Ces points de convergence et de divergence entre les deux cultures mériteraient d'être pris en compte dans le cadre de négociations commerciales par les opérateurs économiques africains et asiatiques.
$\mathrm{P}$ our améliorer le cadre de vie de ses populations et sortir de la pauvreté, l'Afrique doit se repositionner dans le nouvel environnement mondial où l'innovation et la concurrence sont les leitmotiv. Le défi des pays africains est donc l'accès aux différents marchés internationaux et ce d'autant plus que l'Afrique souffre toujours d'une faible intégration dans le marché mondial (voir le rapport de la Banque mondiale sur les perspectives économiques 2005).

Si l'Asie, par son poids démographique, son avancée technologique et le dynamisme de son économie, s’impose désormais comme un acteur important dans le cadre des échanges internationaux, la nécessité d'encourager et de développer les relations de coopération éco-

1. Pour donner une base objective au concept de pays émergents, le CEPH (Centre d'études prospectives et d'informations internationales) a donné trois critères: un niveau de richesse (revenu moyen par tête en 1996 inférieur à $70 \%$ au niveau des pays de 1'OCDE), une participation croissante aux échanges internationaux de produits manufacturés (croissance des exportations de produits manufacturés - y compris les industries agroalimentaires supérieure de $2 \%$ en moyenne par an à la croissance des échanges mondiaux) et l'attraction que ces pays exercent sur les flux internationaux des capitaux » (voir M. Fouquin, 1999). De nombreux pays d'Asie du Sud-Est obéissent à ces critères : Chine, Inde, Indonésie, Malaisie, Corée du Sud, Thaïlande, Taiwan, Singapour, Hong-Kong, Philippines, etc. 
nomique et commerciale entre pays africains et pays asiatiques s'avère primordiale. Dans cette perspective, certains cadres de réflexion et institutions sont à pied d'œuvre (Conférence internationale de Tokyo sur le développement de l'Afrique - TICAD, Forum Afrique-Asie - FAA). Ces institutions œuvrent, avec l'appui des organisations internationales (Nations unies par exemple), pour le renforcement du partenariat entre l'Afrique et l'Asie, le développement des échanges en matière d'expériences de développement entre les deux continents et surtout le développement du secteur privé (considéré comme moteur de croissance dans les deux régions) afin de réduire la pauvreté et d'intégrer les économies africaines dans l'économie mondiale. Or, faut-il le relever, la réussite du partenariat entre individus et pays engagés dans la coopération internationale ne peut et ne doit sous estimer l'importance de la variable culturelle. En effet, les méthodes d'expansion internationale des entreprises comportent un risque culturel (Hofstede, 2001). De même, les négociations internationales sont par nature interculturelles et intersubjectives (Pluchart, 1996).

Toutefois, qu'on s'interroge sur l'impact de la culture dans la façon de gérer les fusions ou les entreprises conjointes internationales (Lee Marks et Mirris, 2000), sur le rôle de la culture dans l'option de l'orientation à court terme ou à long terme des performances des entreprises (Peterson et al., 2002), sur les considérations cross-culturelles d'une généralisation de la théorie de l'agence (Nancy et Scott, 2004), ou encore sur le management des conflits entre cultures en rapport avec l'éthique des affaires (Buller et al., 2000), autant d'aspects qui affectent les relations d'affaires entre individus et nations, l'axe privilégié a toujours été celui de l'Occident et de l'Orient. Aussi, peut-on noter que dans un contexte où l'accent est mis sur l'écoute et la compréhension des différences et où il faut désormais allier la compréhension des êtres humains à la compréhension technique (Breuer et Bartha, 1993) :

- l'Afrique est peu représentée dans les études comparatives en matière de management;

- les données existantes sur l'Afrique ne sont pas suffisamment exploitées (les indicateurs culturels connus à ce jour sur l'Afrique sont encore peu utilisés);

- la culture africaine est parfois évaluée sur la base des anecdotes (Bourgoin, 1984).

C'est dans cette perspective que nous estimons que l'axe Afrique-Asie en termes de compréhension des différences culturelles doit être abordé et visité; la nécessité des relations d'affaires entre l'Afrique et l'Asie se faisant de plus en plus ressentir.

Dans ce travail, nous avons une double préoccupation. D'abord saisir les différences et les similitudes culturelles fondamentales entre cultures africaines et asiatiques (pour la plupart les pays émergents d'Asie du Sud-Est). Ensuite, mettre en évidence leur impact probable dans les relations d'affaires entre Africains et ressortissants des pays émergents d'Asie. Pour atteindre cet objectif nous allons nous appuyer sur la notion de distance culturelle. Les données relatives à l'évaluation des distances culturelles entre l'Afrique et l'Asie seront issues de quelques enquêtes quantitatives (surtout cross-culturelles) menées en Afrique et en Asie. 


\section{I. - ÉVALUATION DES DISTANCES CULTURELLES ENTRE L'AFRIQUE ET L'ASIE}

Pour relever les différences culturelles fondamentales entre l'Afrique au Sud du Sahara et l'Asie du Sud-Est, nous allons nous référer à trois études cross-culturelles: l'enquête IBM de Geert Hofstede (1980, 2001), l'étude CVS (The Chinese Culture Connection, 1988) sur les valeurs cultu- relles chinoises et l'enquête pilote de l'IRIC sur les valeurs culturelles africaines (Africa Value Suvey Module - AVSM (1996-1998)) dirigée par Noorderhaven. C'est la notion de distance culturelle telle que présentée par Grange $(1997)^{2}$ qui sera utilisée dans ce travail pour l'appréciation des distances culturelles entre cultures africaines et est-asiatiques. Pour la mesure des distances culturelles, on peut se référer à l'encadré ci-dessous.

\section{ÉVALUATION DES DISTANCES CULTURELLES}

Pour tenter une évaluation de ce que pourrait être la distance culturelle entre deux nations, Grange ${ }^{3}$ va reprendre les résultats de Hofstede. Il suppose en effet que les quatre indices culturels mis en évidence par Hofstede sont des coordonnées d'un point dans un espace à quatre dimensions. Cet espace peut donc être reporté à 4 axes :

- un axe $\mathrm{m}_{1}$, axe des distances hiérarchiques.

- un axe $\mathrm{m}_{2}$, axe des contrôles de l'incertitude.

- un axe $\mathrm{m}_{3}$, axe d'individualisme.

- un axe $\mathrm{m}_{4}$, axe de la masculinité.

Dans cet espace, qui est l'espace de Geert Hofstede, chaque culture $C^{i}$ peut être représentée par un point de coordonnées:

$-\mathbf{m}_{\mathbf{i} 1}$, indice de distance hiérarchique de $\mathrm{C}^{\mathrm{i}}$.

$-\mathbf{m}_{\mathrm{i} 2}$, indice de contrôle de l'incertitude de $\mathrm{C}^{\mathrm{i}}$.

$-\mathbf{m}_{\mathrm{i} 3}$, indice individualisme de $\mathrm{C}^{\mathrm{i}}$.

$-\mathbf{m}_{\mathbf{i} 4}$, indice masculinité de $\mathrm{C}^{\mathrm{i}}$.

Ainsi, une culture $\mathrm{C}^{\mathrm{i}}$ sera notée $\left(\mathbf{m}_{\mathbf{i} 1}, \mathbf{m}_{\mathrm{i} 2}, \mathbf{m}_{\mathrm{i} 3}, \mathbf{m}_{\mathbf{i} 4}\right)$. De même une culture $\mathrm{C}^{\mathrm{j}}$ sera notée $\operatorname{par}\left(\mathbf{m}_{\mathbf{j} 1}, \mathbf{m}_{\mathbf{j} 2}, \mathbf{m}_{\mathbf{j} 3}, \mathbf{m}_{\mathbf{j} 4}\right)$.

En étendant de façon arbitraire la définition géométrique de distance géométrique entre deux points, Grange va appeler « distance culturelle » (sous-entendu psychologique) la racine carrée des différences entre indices de même signification. Aussi, si on appelle $\mathbf{D}^{\mathrm{ij}}$ la distance entre la culture $\mathrm{C}^{\mathrm{i}}$ et la culture $\mathrm{C}^{\mathrm{j}}$, cette définition se traduit par la formule:

$$
D^{i j}=\left[\left(m^{i 1}-m^{j 1}\right)^{2}+\left(m^{i 2}-m^{j 2}\right)^{2}+\left(m^{i 3}-m^{j 3}\right)^{2}+\left(m^{i 4}-m^{j 4}\right)^{2}\right]^{1 / 2}
$$

2. C'est cette notion de distance culturelle qui a été utilisée par Grange pour mettre en évidence l'impact des différences culturelles dans l'échec de la fusion Volvo-Renault (voir Grange, 1997, p. 105-116).

3. La notion de distance culturelle est aussi utilisée par des auteurs comme Kogut et Singh (1988), article cité par Hofstede (2001, p. 446). 
L'application de la formule [1] présentée dans l'encadré entraîne certains commentaires et suggére quelques remarques et leçons ${ }^{4}$ :

- la distance culturelle ainsi définie est une synthèse possible des quatre dimensions culturelles. Son utilité ne sera validée que par les conséquences pratiques tirées de la formule [1] ;

- la notion de distance culturelle n'a d'intérêt que si les résultats numériques donnés par l'application de la formule [1] rendent compte globalement des différences culturelles ressenties par des groupes d'observateurs de nationalités différentes;

- la distance culturelle telle que définie dépend beaucoup plus de la culture de l'observateur. Aussi peut-on se placer du point de vue de l'observateur de Hofstede, de Bond (The Chinese Culture Connection) ou de l'Africain des travaux de l'RIC;

- la valeur de la distance culturelle ne doit être utilisée que comme signal d'alarme:

- si elle est élevée, il faut sûrement tenir compte dans l'approche du pays avec lequel on veut travailler de l'importance des différences culturelles ainsi mises en évidence. Elle doit impérativement, sous peine de déboires, voire d'échecs, être intégrée dans la stratégie de l'entreprise. Une analyse plus fine au niveau des indices indiquera plus clairement les points sensibles sur lesquels des erreurs pourraient être fatales et les points sur lesquels porter une attention particulière pour être plus efficace ;

- si elle est faible, cela peut vouloir dire que les cultures présentent de nombreux points communs ou de grandes similitudes. Mais cela peut aussi vouloir dire que les diffé- rences ne portent pas sur les mêmes indices. Il faudra donc procéder à un examen minutieux des variables contenues dans les différents indices pour savoir si la globalisation faite en utilisant la notion de distance culturelle n'a pas totalement masqué les différences. Aussi le signal d'alarme n'a-t-il pas une fiabilité à $100 \%$.

Ces différents aspects démontrent la relativité des indicateurs utilisés pour saisir les cultures. Aussi, les distances culturelles serontelles calculées du point de vue de l'observateur européen (dimensions de Hofstede), de l'observateur chinois (The Chinese Culture Connection) et de l'Africain (les dimensions de l'« African Value Survey Module »).

\section{Les distances culturelles du point de vue de l'observateur européen de Hofstede}

Dans ses travaux, Hofstede (1980, 2001) a défini quatre dimensions culturelles:

- La distance hiérarchique (PDI) : cette dimension mesure le degré d'acceptation de l'inégalité dans la répartition du pouvoir entre membres d'une société. Un score élevé sur cette dimension traduit une distance hiérarchique grande et un score moins élevé une faible distance hiérarchique.

- L'individualisme collectivisme (IDV) : cette dimension sert à caractériser les rapports entre l'individu et la société. Ces rapports sont le reflet de la manière de vivre ensemble en société. Un score faible sur cette dimension traduit le collectivisme et un score élevé l'individualisme.

- Masculinité-féminité (MAS) : cette dimension sert à définir la distribution sociale des rôles entre sexes. Dans certaines

4. Pour toutes remarques et leçons sur le calcul des distances culturelles voir le chapitre 3 « Peut-on prévoir l'ampleur des difficultés dues aux différences de culture » de l'ouvrage de Grange (1997). 
sociétés, l'accent est mis sur la réussite matérielle et la démonstration de la force et de l'autorité (sociétés masculines). Dans d'autres, on privilégie la modestie et tendresse et on s'intéresse à la qualité de la vie (sociétés féminines). Un score élevé sur cette dimension traduit l'orientation vers le pôle masculin et un score faible l'orientation vers le pôle féminin.

- Le contrôle de l'incertitude (UAI) : cette dimension mesure le degré d'inquiétude des habitants face aux situations ambiguës, inconnues ou incertaines. Les valeurs de cet indice se situent entre 0 pour le degré de contrôle le plus faible et 100 pour le degré le plus fort.

Les scores des pays africains et asiatiques sur ces différentes dimensions sont donnés dans le tableau 1.

Dans le travail de Hofstede (1980, 2001), l'Asie du Sud-Est est représentée par 12 pays, tandis que les pays africains sont regroupés en deux régions (Afrique de l'Ouest et Afrique de l'Est). Les scores d'autres pays asiatiques (Chine, Vietnam, Bangladesh) sur les quatre dimensions sont donnés par Hofstede (2001) sur la base des estimations. De même, on note aussi l'évaluation de deux autres pays francophones d'Afrique sur les 4 dimensions à savoir le Cameroun $^{5}$ et la Côte d'Ivoire ${ }^{6}$.

Le tableau 2 donne une évaluation des distances culturelles entre les pays africains au Sud du Sahara et ceux de l'Asie du Sud-Est. $\mathrm{Au}$ regard de ces résultats, certaines remarques peuvent être faites :

- les distances culturelles entre pays africains et pays asiatiques concernés sont soit faibles, soit moyennes ;

- de manière générale, les pays africains sont beaucoup plus proches des pays comme l'Indonésie, la Thaïlande, le Pakistan et Taiwan. Ce résultat était attendu dans la mesure où les résultats issus de l'analyse hiérarchique effectuée par Hofstede regroupent dans un même ensemble (groupe 3) les pays africains et ceux ci-dessus mentionnés.

\begin{tabular}{|l|c|c|c|c|l|l|l|l|c|}
\hline \multicolumn{10}{|c|}{ Tableau 1 } \\
\hline \multicolumn{10}{|c|}{ INDICES DES PAYS AFRICAINS ET ASIATIQUES SELON HOFSTEDE } \\
\hline Pays & PDI & UAI & IDV & MAS & \multicolumn{1}{|c|}{ Pays } & PDI & UAI & IDV & MAS \\
\hline Indonésie & 78 & 48 & 14 & 46 & Inde & 77 & 40 & 48 & 56 \\
\hline Thaïlande & 64 & 64 & 20 & 34 & Singapour & 74 & 8 & 20 & 48 \\
\hline Pakistan & 55 & 70 & 14 & 50 & Hong-Kong & 68 & 29 & 25 & 57 \\
\hline Taiwan & 58 & 69 & 17 & 45 & Afrique de l'Est & 64 & 52 & 27 & 41 \\
\hline Corée du Sud & 60 & 85 & 18 & 39 & Afrique de l'Ouest & 77 & 54 & 20 & 46 \\
\hline Philippines & 94 & 44 & 32 & 64 & Cameroun & 52 & 76 & 21 & 35 \\
\hline Malaisie & 104 & 36 & 26 & 50 & Côte d'Ivoire & 68 & 48 & 20 & 34 \\
\hline Chine & 80 & 30 & 20 & 66 & Vietnam & 70 & 30 & 20 & 40 \\
\hline
\end{tabular}

5. Les travaux sur le Cameroun sont issus d'une enquête menée auprès de plus 300 étudiants travailleurs, les scores sur les dimensions sont calculés sur la base des formules issues du VSM 94 (Value Survey Module).

6. Les résultats sur la Côte d'Ivoire sont issus des travaux de Bourgoin, (1984). 


\begin{tabular}{|l|c|c|c|c|}
\hline \multicolumn{5}{|c|}{ Tableau 2 } \\
CALCUL DES DISTANCES CULTURELLES ENTRE PAYS ASIATIQUES \\
ET AFRICAINS \\
\hline & Afrique de l'Est & Afrique de l'Ouest & Cameroun & Côte d'Ivoire \\
\hline Indonésie & 20 & 8 & 40 & 17 \\
\hline Thaïlande & 16 & 20 & 18 & 16 \\
\hline Pakistan & 26 & 28 & 29 & 31 \\
\hline Taiwan & 21 & 24 & 23 & 26 \\
\hline Corée du Sud & 34 & 36 & 27 & 38 \\
\hline Philippines & 39 & 29 & 58 & 42 \\
\hline Malaisie & 44 & 33 & 63 & 42 \\
\hline Inde & 31 & 33 & 47 & 38 \\
\hline Singapour & 46 & 46 & 66 & 43 \\
\hline Hong-Kong & 28 & 29 & 50 & 30 \\
\hline
\end{tabular}

De façon générale, on peut noter que l'Afrique partage avec l'Asie du Sud-Est, lorsqu'on se réfère à l'observateur de Hofstede, une distance hiérarchique longue ou moyenne et une mentalité plus communautaire.

On note aussi que l'Afrique et l'Asie du Sud-Est s'éloignent quelque peu sur les dimensions masculinité et contrôle de l'incertitude. C'est d'ailleurs les scores obtenus par les pays asiatiques sur ces deux dimensions qui sont la cause principale des disparités constatées entre eux (Bollinger et Hofstede, 1987, p. 171). Les grandes disparités entre cultures africaines et asiatiques apparaissant toutefois au niveau du contrôle de l'incertitude où certains pays comme la Corée du Sud, Taiwan et la Thaillande enregistrent des scores assez élevés. Le score moyen comme celui du Cameroun sur la dimension distance hiérarchique révèle tout simplement la tendance égalitaire déjà constatée dans de nombreux groupes ethniques camerounais (Laburthe-Tolra; 1981, Mveng, 1984, Balandier, 1982). De même, le score élevé du Cameroun sur la dimension contrôle de l'incertitude peut révéler la propension au respect des manuels de procédure et aux règles dans les organisations africaines (Henry, 1991, p. 447-473) Intéressons-nous cette fois au calcul des distances culturelles selon l'observateur de Bond (l'observateur chinois).

\section{Les distances culturelles du point de vue de l'observateur asiatique de Bond}

Les travaux de Bond introduisent le biais oriental dans les études cross-culturelles. L'enquête cross-culturelle basée sur les valeurs culturelles chinoises: «The Chinese Value Connection » a été menée dans 23 pays dont 2 pays africains (Zimbabwé et Nigéria) et 10 pays asiatiques. L'analyse 
des données de cette enquête a permis de dégager 4 facteurs $^{7}$ : le facteur I (CVS I) dénommé «Integration », le facteur II (CVS II) dénommé « Confucian dynamism », le facteur III (CVS III) dénommé «Human-heartedness » (« Chaleur humaine ») et le facteur IV (CVS IV) dénommée « Moral discipline ».

Entre facteurs CVS et facteurs IBM, Hofstede $(1994,2001)$ a mis en évidence un certain nombre de corrélations et correspondances $^{8}$. D'abord, une forte distance hiérarchique (IBM) représente un besoin de dépendance par rapport à des personnes plus puissantes. Le facteur CVS correspondant la «Discipline morale » est décrit par les valeurs relatives à la limitation des désirs, à la modération, à la recherche du juste milieu, au désir de rester désintéresser et pur. À l'opposé, une faible distance hiérarchique traduit un besoin d'indépendance. Le facteur CVS correspondant renvoie aux valeurs relatives à la flexibilité et à la prudence. Ensuite, l'individualisme (IBM) cor- respond à l'un des pôles du facteur CVS qui est « l'intégration »; les variables qui composent ce facteur sont relatives à la tolérance, à la recherche de l'harmonie, au refus de la concurrence, au désir d'avoir un véritable ami intime, au désir de compter pour le groupe, à la résignation à son sort, au désir de solidarité avec les autres et à la nécessité d'être conservateur. Le pôle opposé, le collectivisme, correspond à d'autres valeurs de l'étude CVS comme: la piété filiale, la chasteté pour les femmes, le patriotisme. Enfin, à la masculinité (IBM) correspond le facteur CVS « Chaleur humaine ». Au pôle de la masculinité vont correspondre les valeurs relatives à la courtoisie, à la gentillesse et à la compassion. $\mathrm{Au}$ pôle féminin, c'est le patriotisme (comme pour le collectivisme) et la droiture qui seront les valeurs prédominantes.

Les indices culturels des pays asiatiques et africains sont donnés dans le tableau 3 cidessous.

\begin{tabular}{|l|c|c|c|c|l|c|c|c|c|}
\hline \multicolumn{10}{|c|}{ Tableau 3 } \\
INDICES DES PAYS AFRICAINS ET ASIATIQUES \\
SELON L'OBSERVATEUR DE BOND (CVS) \\
\hline Pays & CVS1 & CVS2 & CVS3 & CVS4 & \multicolumn{1}{|c|}{ Pays } & CVS1 & CVS2 & CVS3 & CVS4 \\
\hline Japon & 78 & 80 & - & 24 & Inde & $\mathbf{1 5}$ & 61 & $\mathbf{5 4}$ & 42 \\
\hline Thaïlande & $\mathbf{4 3}$ & 56 & $\mathbf{6 3}$ & 37 & Singapour & $\mathbf{5 3}$ & 48 & $\mathbf{7 5}$ & 25 \\
\hline Pakistan & $\mathbf{1 7}$ & - & $\mathbf{5 9}$ & 61 & Hong-Kong & $\mathbf{4 9}$ & 96 & $\mathbf{8 6}$ & 34 \\
\hline Taïwan & $\mathbf{3 7}$ & 87 & $\mathbf{6 6}$ & 39 & Bangladesh & - & 40 & $\mathbf{5 9}$ & 21 \\
\hline Corée du Sud & $\mathbf{5 5}$ & 75 & $\mathbf{6 1}$ & 80 & Nigéria & $\mathbf{3 9}$ & $\mathbf{1 7}$ & $\mathbf{7 5}$ & $\mathbf{3 1}$ \\
\hline Philippines & 62 & 20 & $\mathbf{8 7}$ & 100 & Zimbabwé & $\mathbf{5 0}$ & $\mathbf{2 5}$ & $\mathbf{8 1}$ & $\mathbf{2}$ \\
\hline
\end{tabular}

7. Pour plus de détails sur les différentes valeurs culturelles qui constituent les différentes dimensions voir l'article «Chinese values and the search for culture-free dimensions of culture », Journal of Cross-Cultural Psychology, 18, 1987, p. 143-164.

8. Pour plus de détails voir Hofstede (2001, p. 351-372). 


\begin{tabular}{|l|c|c|}
\hline \multicolumn{2}{|c|}{ Tableau 4 } \\
CALCUL DES DISTANCES CULTURELLES ENTRE PAYS ASIATIQUES \\
ET AFRICAINS SELON L'OBSERVATEUR DE BOND (CVS) \\
\hline Nigéria & Zimbabwé \\
\hline Japon & 81 & 81 \\
\hline Philippines & 74 & 99 \\
\hline Corée du Sud & 79 & 95 \\
\hline Singapour & 35 & 33 \\
\hline Hong-Kong & 80 & 78 \\
\hline Thailande & 41 & 74 \\
\hline Taiwan & 71 & 75 \\
\hline Pakistan & 43 & 70 \\
\hline Inde & 55 & 60 \\
\hline Bangladesh & 49 & 51 \\
\hline
\end{tabular}

Les distances culturelles entre pays africains et asiatiques sont données dans le tableau 4.

On peut remarquer que :

- Les distances culturelles entre les pays africains et les pays asiatiques comme le Singapour, la Thaïlande et le Pakistan (particulièrement avec le Nigéria) sont moins grandes. Par contre, ces distances sont plus remarquables avec les pays comme les Philippines, la Corée du Sud, Hong-Kong et Taiwan.

- Les différences culturelles sont beaucoup plus accentuées pour la dimension «Confucian dynamism » où les scores des pays africains sont faibles et ceux des pays asiatiques plus élevés 9 .

- Ces différences sont moins marquées, quoique à des degrés divers, pour les autres dimensions où on peut remarquer que les pays africains concernés par l'enquête se rapprochent beaucoup plus :

- des pays comme: la Corée du Sud, Singapour, Hong-Kong et la Thaillande sur la dimension «Intégration »;

- des pays comme: les Philippines, Singapour, Hong-Kong, la Corée du Sud et Taiwan sur la dimension « Human-heartedness »;

- des pays comme: l'Inde, Taiwan, Thaïlande, Hong-Kong et Singapour sur la dimension « Moral discipline » particulièrement pour le Nigéria, le score du Zimbabwé se rapprochant beaucoup plus sur cette dimension à celui des pays anglosaxons (Angleterre, États-Unis, Suède, Nouvelle Zélande et Canada).

9. Le score du Cameroun calculé pour cette dimension est aussi faible, ce qui peut suggérer une orientation à court terme pour les pays africains. 
De la même manière que dans les cas précédents intéressons-nous à présent aux différences culturelles entre l'Afrique et l'Asie du Sud-Est du point de vue de l'observateur africain.

\section{Les distances culturelles du point de vue de l'observateur africain}

L'étude sur les valeurs culturelles africaines est inspirée de celle sur les valeurs culturelles chinoises (CVS) menée par Bond. L'instrument utilisé pour cette enquête a été élaboré sur la base de la méthode Delphi. Ainsi, un certain nombre d'opinions d'experts africains sur les valeurs culturelles africaines a permis de mettre au point un questionnaire comportant 82 variables. Ce questionnaire a été présenté aux étudiants en management et en économie dans 14 pays (6 pays africains, 4 européens, 2 asiatiques, 1 d'Amérique du Sud et les États-Unis).

L'analyse des résultats issus de cette enquête a permis de dégager six facteurs pour la détermination de huit dimensions. Trois dimensions étant issues du premier facteur. Les dimensions ainsi identifiées sont: la dimension «Bonté humaine » (« Human goodness ») (HG), la dimension «Règles et hiérarchie » (« Rules and hierarchy ») $(\mathrm{RH})$, la dimension « Importance de la religion » («Importance of religion ») (IR), la dimension « Sagesse ancestrale » (« Traditional wisdom ») (TW), la dimension «Partage » (« Sharing ») $(\mathrm{SH})$, la dimension «Jalousie» (« Jealousy ») (JE), la dimension «Collectivisme» («Collestivism ») (CO) la dimension « Responsabilité sociale » («Societal responsability »). Voici par ailleurs une description synoptique des ces huit dimensions ${ }^{10}$.

\section{La dimension "Bonté humaine » ( Human Goodness »)}

L'examen des variables contenues dans cette dimension amène à considérer deux phénomènes concomitants: une vision positive de l'individu et la conviction selon laquelle cette vision positive serait inhérente à la conception du travail dans les organisations. Aussi, un score élevé sur cette dimension serait la traduction d'une vision optimiste de la nature humaine et la conviction que cette vision serait prédominante dans les principes organisationnels. Un score faible, une vision pessimiste de la nature humaine et une représentations conséquentes des principes d'organisation.

\section{Règles et hiérarchie («Rules and Hierarchy»)}

Les trois items très représentatifs de cette dimension et qui constituent ses éléments fondamentaux sont relatifs aux règles, aux procédures, et aux rapports avec les supérieurs. Un score faible indique que les règles et la hiérarchie sont moins importantes. Cette dimension est fortement corrélée et de façon significative aux dimensions « distance hiérarchique » et « collectivisme-individualisme » de Hofstede.

\section{Importance de la religion ( Importance of Religion »)}

Les variables contenues dans cette dimension opposent les valeurs religieuses aux valeurs hédonistes. Un score élevé sur cette dimension indique une plus grande importance accordée aux valeurs religieuses et non aux valeurs hédonistes, un score faible traduit le contraire. 


\section{La sagesse traditionnelle ( Traditional Wisdom »)}

Les variables qui constituent cette dimension peuvent être considérées comme relevant de la sagesse traditionnelle africaine: esprit de consensus, hospitalité et respect de la tradition. Un score élevé sur cette dimension indique que les répondants acceptent les points de vue traditionnels de la sagesse, font preuve d'hospitalité et approuvent l'esprit de consensus.

\section{Le partage ( Sharing »)}

Les variables qui servent à caractériser cette dimension mettent en avant, non seulement l'idée de partage des richesses, mais aussi, celle selon laquelle, les personnes disposant de ressources, de talent, d'habileté doivent obligatoirement venir en aide à celles qui sont dans le besoin (devoir d'assistance). Un score élevé sur cette dimension traduit la forte tendance pour les individus au partage des richesses et à l'assistance aux autres.

\section{Jalousie ( Jealousy »)}

Les items fortement corrélés à cette dimension expriment une vision négative du com- portement humain: jalousie, ambition, peur d'être ridiculisé, tromperie. Un score élevé sur cette dimension peut traduire la crainte de s'écarter de la société ou encore une forte sensation de «jalousie ».

\section{Collectivisme ( Collectivism»)}

Certains items relatifs à cette dimension suggèrent l'idée de collectivisme ou d'esprit communautaire et d'autres renvoient à l'idée d'intérioriser ses pensées et ses ambitions et aussi au fatalisme. Toutefois, ce facteur ne présente aucune corrélation avec la dimension individualisme-collectivisme de Hofstede ou encore avec d'autres dimensions comme celles de Schwartz allant dans ce sens.

\section{Responsabilité « sociétale 》(«Societal Responsability»)}

Les deux items fortement corrélés à cette dimension traduisent la responsabilité sociale des individus face à la société: responsabilité du citoyen pour la construction de leur société et nécessité de la collaboration entre individus de groupes ethniques différents pour la réalisation du bien être de la société.

\begin{tabular}{|l|c|c|c|c|c|}
\hline \multicolumn{10}{|c|}{ Tableau 5 } \\
\multicolumn{10}{|c|}{ INDICES DES PAYS AFRICAINS ET ASIATIQUES SUR LES DIMENSIONS } \\
CULTURELLES AFRICAINES \\
\hline \\
\hline
\end{tabular}


Le tableau 5 ci-avant donne les scores des différents pays africains et asiatiques sur ces facteurs (Afrique du Sud non compris, l'enquête dans ce pays n'ayant concerné que la population blanche).

Les distances culturelles entre pays africains et pays asiatiques sur les dimensions culturelles africaines sont données dans le tableau 6 ci-dessous.

De façon générale, on peut noter que:

- les distances culturelles entre pays africains et pays asiatiques sur les dimensions africaines sont élevées. Toutefois, la Malaisie se rapproche des pays africains sur de nombreuses dimensions contrairement à Hong-Kong ;

- l'Afrique et l'Asie de l'Est partagent en commun les mêmes caractéristiques en ce qui concerne la considération de la hiérarchie et le degré de vie communautaire ;

- la grande disparité entre l'Afrique et l'Asie apparaît au niveau de la dimension « Sagesse traditionnelle », dimension qui est par ailleurs corrélée négativement et de façon significative à la dimension «Confuceen dynamism » (Noorderhaven et Tidjani, 2001) ;

- l'Afrique et l'Asie diffèrent aussi du point de vue de la conception de la religion. Si la
Malaisie se rapproche de l'Afrique sur la dimension « importance de la religion », parce que la religion pratiquée est l'islam, tel n'est pas le cas de Hong-Kong qui partage avec d'autres pays d'Asie du Sud-Est le shintoïsme, l'hindouisme, le boudhisme et le taoïsme et autres religions asiatiques. Des études ici présentées, l'Afrique et l'Asie du Sud-Est présentent avec plus clarté deux points communs: distance hiérarchique moyenne ou grande et fort degré de vie communautaire. Ces deux entités se rapprochent aussi sur les valeurs relatives à la féminité, à la chaleur humaine et au partage. Ces travaux font aussi apparaître deux points de divergence: l'Afrique n'est pas imprégnée des enseignements de Confucius qui ont fait le progrès des pays de l'Asie du Sud-Est et les pays de l'Asie du Sud-Est connaissent mal les valeurs censées soustendre le point de vue de la sagesse africaine.

En outre, une analyse de certaines variables de l'enquête cross-culturelle menée par l'IRIC, enquête qui, rappelons le, s'appuie sur les valeurs culturelles africaines, a permis de mettre en évidences les ressemblances et les différences entre pays africains et pays asiatiques sur les six

\begin{tabular}{|l|c|c|}
\hline \multicolumn{3}{|c|}{ Tableau 6 } \\
\multicolumn{2}{|c|}{$\begin{array}{c}\text { DISTANCES CULTURELLES ENTRE PAYS AFRICAINS ET PAYS D'ASIE } \\
\text { DU SUD-EST SUR LES DIMENSIONS AFRICAINES }\end{array}$} \\
\hline & Hong-Kong & Malaisie \\
\hline Cameroun & 118 & 115 \\
\hline Sénégal & 150 & 138 \\
\hline Ghana & 121 & 112 \\
\hline Tanzanie & 129 & 111 \\
\hline Zimbabwé & 131 & 115 \\
\hline
\end{tabular}


dimensions de l'orientation culturelle des peuples telles que décrites par les anthropologues: la nature de l'être humain (bon ou mauvais), la relation avec la nature et avec le monde, les relations avec les autres, l'orientation de l'activité (être ou faire), l'orientation spatiale et l'orientation temporelle. Ici, on a encore noté que la grande différence entre l'Afrique et l'Asie se situe au niveau de la conception de la nature humaine, de la relation de l'individu avec la nature (soumission vs. harmonisation), de la conception du temps (prédilection du passé ou affection pour le futur) et de l'orientation de l'activité humaine (l'orientation « être » étant beaucoup plus la caractéristique des sociétés africaines).

Ces différences et ces points communs, déjà relevés, peuvent avoir un impact dans les relations d'affaires entre les Africains et les habitants du Sud-Est asiatique.

\section{II. - NÉGOCIATION D'AFFAIRES ET SYNERGIE CULTURELLE DANS L'AXE AFRIQUE-ASIE DU SUD-EST}

$\mathrm{Au}$ cours des dernières décades du $\mathrm{XX}^{\mathrm{e}}$ siècle, le processus de l'internationalisation ou de l'expansion internationale a été dominé par les opérations d'investissements directs. En Asie du Sud-Est, surtout après la crise asiatique de 1997 , on a noté que les fusions avec les entreprises nationales ou les acquisitions par les entreprises internationales (fusions et acquisitions) ont connu une progression spectaculaire. Si lors des opérations d'internationalisation des entreprises les préoccupations financières sont prioritaires, force est de constater que les facteurs culturels influencent, parfois de manière décisive, la réussite ou l'échec des joint-ventures, acquisitions, fusions et autres opérations d'insertion dans les marchés extérieurs. En effet, les facteurs culturels se trouvent à l'œuvre à divers niveaux du processus de négociations et conditionnent d'abord les méthodes d'expansion internationale et ensuite la chance de survie de l'affaire. Au-delà de la prise en compte $\mathrm{du}$ facteur culturel dans le processus de négociation, il faut d'abord relever qu'élaborer dans le contexte actuel un cadre conceptuel de négociation internationale est un exercice qui oblige à avoir recours à une approche holiste.

\section{Quelques éléments caractéristiques du contexte de négociation des affaires}

Le contexte actuel, caractérisé par l'influence réciproque des affaires publiques et privées, l'effacement des préjugés nationaux, la permanence des menaces de désordre et de rupture, rend non seulement nécessaire l'exercice sagace et de longanimité de la négociation; mais aussi, oblige à renouveler la vision de la négociation internationale dans ses principes et méthodes (Plantey, 2002, p. 7 et 24).

Le champ d'étude du processus de négociation se caractérise d'abord par la cohabitation des approches théoriques et pratiques. Or, il est désormais nécessaire de dépasser cette vision dichotomique de la négociation. C'est pourquoi différents auteurs insistent, dans la conceptualisation des modèles de négociation, sur la nécessité d'allier les analyses théoriques s'appuyant sur des concepts et autres théories et les considérations pratiques puisant dans les expériences accumulées par les professionnels (Arvind et Habib, 1996 ; Yadong, 1999 ; 
Rojot, 1994). À ce sujet, J. Rojot note qu' « une théorie pertinente fournit un cadre conceptuel à son utilisateur, un guide à la compréhension, une carte cognitive qui peut être utilisée pour analyser le concret et comprendre mieux ce qui pourrait et devrait être fait dans une situation pratique réelle et donnée ».

La négociation se caractérise aussi par les nombreuses dimensions et éléments qu'elle doit prendre en compte. En effet, si dans le cadre de la négociation internationale on est d'avis que la connaissance des déterminants, des processus et des résultats peut aider le manager à adopter les stratégies de collaboration garantissant le maximum de bénéfice (Yadong, 1999), il apparaît aussi que le processus de négociation est luimême influencé par de nombreuses variables contextuelles (le pluralisme légal, le pluralisme politique, les instabilités et les changements, les différences idéologiques, les différences culturelles, les différents stakeholders, etc.) (Arvind et Habib, 1996) et d'autres facteurs relatifs aux antécédents environnementaux, organisationnels et individuels, à la concurrence et aux conséquences de la négociation (Yadong, 1999).

Cette situation appelle donc à prendre en considération les apports de différentes disciplines et sous-disciplines ainsi que de différentes écoles de pensée. À ce sujet, comme nous le rappelle Rojot, Zartman distingue en fonction de l'approche analytique utilisée sept écoles auxquelles il faut ajouter une huitième qui emprunte transversalement des éléments méthodologiques aux sept précédentes: historique, contextuelle, structurelle, stratégique, par types de personnalités, par capacités comportementales, par variables processuelles et procédurales; cette dernière utilisant l'expérimentation et la simulation (op. cit. p. 2). Les disciplines sollicitées dans le cadre de la conceptualisation du processus de négociation vont de l'économie aux sciences de gestion en passant par la sociologie, l'anthropologie et la psychologie sociale.

La multiplicité des variables et dimensions à prendre en compte, la nécessité d'allier théorie et pratique ont amené Rojot à proposer trois niveaux de conceptualisation de la notion de négociation. De l'avis de cet auteur, «Au premier niveau, il est nécessaire de comprendre l'environnement tel qu'il se présente et tel qu'il va structurer la négociation, c'est le domaine des concepts, de la compréhension et de l'analyse, où se forme le rapport de pouvoir de négociation. Le deuxième passe de la structure au processus. C'est le domaine de la maîtrise des outils techniques, des procédures, où s'élaborent la stratégie et les tactiques. Le troisième niveau est celui du face-à-face d'individus en présence. C'est le domaine des attitudes et du comportement interpersonnel, où se créent les styles de négociation et les modes de gestion des situations. » (op. cit., p. 3).

Notre travail, que nous pouvons situer au premier niveau de la conceptualisation du processus de négociation de Rojot, aura des répercussions sur l'ensemble des opérations liées aux méthodes d'expansion internationales des entreprises compte tenu du fait que les valeurs culturelles peuvent se retrouver à l'œuvre à tous les niveaux du processus de négociation. 


\section{Facteurs culturels et méthodes d'expansion internationales des entreprises}

De façon générale, on peut distinguer avec Hofstede (2001) cinq méthodes d'expansion internationale des entreprises: la création des nouvelles filiales, l'acquisition d'une entreprise étrangère, la fusion internationale, l'entreprise conjointe (« jointventure ») internationale et l'alliance stratégique internationale.

Partant du fait que dans ces opérations d'internationalisation les échanges culturels jouent un rôle primordial, puisque entrent en jeu ici non seulement les cultures nationales mais aussi les cultures d'entreprise (Hofstede, 1994, p. 288), le risque culturel serait donc fonction de la méthode d'expansion. Voici comment Hofstede présente le risque culturel associé à chaque méthode d'expansion:

- la création de nouvelles entreprises, qui consiste à envoyer un expatrié ou une petite équipe monter de toute pièce une filiale à l'étranger, présente un risque culturel limité. Le taux de réussite dans cette méthode d'expansion est très élevé (Hofstede cite le cas d'IBM) ;

- l'acquisition d'une entreprise. Dans cette méthode d'expansion l'entreprise est achetée en bloc, il faut donc intégrer d'un seul coup dans l'entreprise existante sa propre culture et les éléments de la culture du pays. On peut donc concevoir ici que le risque culturel soit énorme et les chances de réussite moins fréquentes;

- la fusion internationale: cette méthode ressemble à la précédente, à cette différence que les partenaires sont de taille ou d'importance sensiblement égale. Le risque culturel est aussi énorme et le taux de réussite selon Hofstede (1994) oscillerait autour de $25 \%$;

- l'entreprise conjointe: c'est la création d'une nouvelle unité par la mise en commun de ressources par deux ou plusieurs parties. Le risque culturel est moins élevé que dans le cas précédent. De l'avis de Hofstede, les entreprises conjointes dans lesquelles l'un des partenaires prend en charge la totalité de la gestion ont un taux de réussite plus élevé qu'en cas de partage de gestion;

- l'alliance stratégique internationale: c'est la méthode la plus prudente de s'internationaliser sans créer une nouvelle entité. Les partenaires décident de collaborer sur des produits et/ou des marchés précis pour leur bénéfice mutuel. De l'avis de Hofstede, comme les risques sont limités à un projet précis, c'est la façon la plus sûre d'apprendre à se connaître et aucune des deux parties ne se sent menacée.

Le deuxième forum Asie-Afrique tenu à Bangkok (Thailande) du 11 au 13 juin 1997, en recommandant de renforcer les relations commerciales et les flux d'investissement, a conseillé la promotion des investissements étrangers et la mise au point d'opération d'association. À notre avis, pour la réussite de telles opérations, il faut aller plus en avant en soulignant les risques culturels associés à chaque type d'opération et en proposant les actions à entreprendre pour une meilleure compréhension entre africains et asiatiques. Sur ce point, Hofstede nous éclaire à nouveau en présentant quelques travaux où la distance culturelle entre pays et les dimensions culturelles (UAI, PDI, MAS, IDV, LTO) sont corrélées au taux de réussite des différentes méthodes d'expansion. 
En effet, note Hofstede, entre 1980 et 1990 , un certain nombre de publications étaient consacrées au choix du mode de pénétration du marché extérieur (choix d'une méthode d'internationalisation). L'examen de ces différentes publications met en évidence l'impact des distances culturelles et des dimensions UAI, MAS, PDI, LTO et IDV sur le choix du mode de pénétration des marchés extérieurs et le taux de chance de survie ou de réussite des différentes structures créées. Trois cas relatifs au marché chinois Tse, Pan et $\mathrm{Au}^{11}$ (1997), Luo ${ }^{12}$ (1999, p. 519) et $\operatorname{Pan}^{13}$ (1996) soulignent l'impact de la dimension PDI et de la distance culturelle dans la pénétration du marché chinois.

Le constat général fait par Hofstede après examen de toutes ces publications indique que la distance culturelle est un facteur important qui influence tant le choix que le taux de réussite des méthodes d'internationalisation. Ensuite, les différences entre PDI et UAI ont une emprise notable sur ce type d'opération. En tout état de cause, il n'existe aucune règle simple applicable entre secteurs, pays et industries.

Les distances culturelles ici calculées, nous indiquent que du point de vue de l'observateur de Hofstede ou européen, L'Afrique et l'Asie du Sud-Est peuvent réussir dans les relations d'affaires tout en mettant un accent particulier pour comprendre leur différence sur le contrôle de l'incertitude. Du point de vue de l'observateur asiatique et africain, les distances culturelles nous indi- quent que l'Afrique et l'Asie s'éloignent sur la dimension orientation à court ou à long terme et sur les considérations qui guident la sagesse en Afrique. Aussi convientil de prendre en compte ces différents aspects dans le cadre des opérations d'internationalisation des entreprises entre l'Afrique et l'Asie du Sud-Est.

Abondant dans le sens des travaux précédents, Grange souligne que deux éléments culturels fondamentaux influencent la conquête des grands contrats internationaux: la valeur de l'indice de distance hiérarchique de la culture nationale du client qui permet une approche raisonnée de décision du client et la valeur de l'indice de l'individualisme qui aide à définir une stratégie fondée sur la réalité culturelle des relations interpersonnelles qui tissent la trame du contexte commercial local et dans lequel il faut s'immiscer (op. cit., p. 190). Toujours selon Grange (op. cit., p. 191), la force de l'argument du commerçant international doit prendre en compte: l'indice du contrôle de l'incertitude, l'indice de la masculinité et l'indice de l'orientation à long terme ou à court terme. S'agissant de ce dernier indice, Grange mentionne que dans les pays à fort indice d'orientation à long terme comme ceux de l'Asie du SudEst, « on trouve dans la valeur élevée de cet indice la traduction de l'orientation de la culture du pays vers les règles pratiques (la Vertu) plus que vers des constructions théoriques (la Vérité). Il est certain que dans un tel contexte culturel, il faudra privilégier les

11. Le travail de Tse, Pan et Au (1997) porte sur l'analyse de 3000 opérations étrangères effectuées en Chine allant des simples opérations d'exportation à l'acquisition des entreprises (voir Hofstede, 2001, p. 447).

12. Luo (1999) a enquêté auprès de 96 entreprises étrangères basées en Chine. Il trouve que la distance culturelle entre les partenaires a un impact négatif sur la performance de l'entreprise. (voir Hofstede, 2001, p. 447).

13. Pan (1996) analyse plus de 4000 entreprises étrangères établies en Chine entre 1979 et 1992 et met en évidence le rapport entre distance culturelle et mode de participation dans l'affaire (voir Hofstede, p. 458). 
arguments à caractère pragmatique » (p. 193). L'acteur africain engagé dans les négociations commerciales avec les opérateurs économiques asiatiques doit donc comprendre que les valeurs liées à l'orientation à long terme, qui correspondent difficilement à la mentalité africaine, sont à la base du pragmatisme chinois dans les relations d'affaires.

L'influence de la culture relève aussi du processus même de la négociation.

\section{La négociation internationale dans l'axe Afrique Asie du Sud-Est}

Dans le cadre des négociations internationales, « on ne peut faire le postulat de valeurs et d'objectifs communs, chaque joueur ne reconnaît que ses propres règles de jeu » (Hofstede, 1994, p. 286). Aussi, de l'avis de Adler, « la négociation est le processus où deux ou plusieurs partenaires, qui partent de besoins et de points de vue divergents essayent d'en venir à une entente sur les questions d'intérêt commun ». Dans le cadre des négociations internationales, les partenaires sont généralement de nationalités et de cultures différentes. C'est donc ainsi qu'apparaît le caractère interculturelle de toute négociation internationale. Or, faut-il le relever, il y a autant de façons de négocier qu'il existe de culture (Adler, 1994, p. 225). De l'avis de Adler, le succès de la négociation internationale repose sur trois grands facteurs: les caractéristiques de chacun des négociateurs en présence, le contexte dans lequel se déroule la négociation et la stratégie et les tactiques mises en œuvre au cours des négociations. Aussi, le bon négociateur choisit-il les stratégies et les tactiques en fonction des acteurs et du contexte (Adler, p. 201).
Abondant dans le même sens, Hofstede cite cinq caractéristiques communes à tout type de négociation interculturelle: deux ou plusieurs parties en conflit d'intérêt, un désir commun d'entente dans l'espoir de tirer un gain de cette entente, l'indétermination de l'issue, les moyens de contrôle dont disposent les parties, le contrôle et la structure de prise de décision chez chacun des partenaires en présence.

Toujours de l'avis de Hofstede, en matière de négociation internationale, les négociateurs peuvent respecter différentes règles de jeu: la nature du contrôle et de la structure de prise de décision chez les différents protagonistes, les individus impliqués dans le processus de prise de décision ainsi que leur pouvoir de décision, les raisons de croire ou de ne pas croire au comportement affiché par le négociateur en face (une certaine de confiance est indispensable si on veut réussir la négociation), la tolérance de l'ambiguïté durant le processus de négociation, les besoins émotionnels du négociateur.

Les dimensions culturelles affectent donc ces différentes règles. La première est affectée par la distance hiérarchique, la seconde par le contrôle de l'incertitude, la troisième par la dimension individualisme collectivisme et la quatrième par la dimension masculinité-féminité. On doit aussi souligner que la dimension de l'orientation à court ou à long terme influence le degré de persévérance avec lequel les buts recherchés sont poursuivis et les sacrifices supportés. Aussi entre les ressortissants de l'Asie du Sud-Est et ceux de l'Afrique, le processus de négociation peut ne pas aboutir si on tient en médiocre estime les différences culturelles liées au contrôle de l'incertitude et à l'orientation à long ou court terme. 
Le contexte Est-asiatique présente un certain nombre de spécificités. Par exemple L. Pye, en soulignant les spécificités de la culture chinoise, culture qui imprègne la majorité des pays d'Asie du Sud-Est, fait quelques recommandations aux étrangers qui veulent entrer en relation d'affaires avec les Chinois ${ }^{14}$. En tout état de cause, il est question à travers ces recommandations et de l'avis de Lucian Pye de discerner les différences culturelles et de les comprendre sans essayer de s'intégrer à la culture de l'autre.

S'agissant en définitive des principes pouvant guider les négociations d'affaires entre les Africains et les ressortissants d'Asie du Sud-Est, nous avons remarqué que:

- Les distances culturelles entre l'Afrique et l'Asie, du point de vue de l'observateur de Hofstede, étaient faibles (généralement inférieures à 40, minimum exigé par Grange pour tirer la sonnette d'alarme). Toutefois, une grande attention doit être portée quant aux différences relatives au contrôle de l'incertitude.

- Du point de vue de l'observateur de l'Orient, c'est-à-dire de Bond, les distances culturelles entre l'Afrique et l'Asie du SudEst sont assez élevées. La différence importante étant celle de la dimension de l'orientation à court terme ou à long terme. À ce niveau l'Africain qui négocie avec un ressortissant des pays émergents d'Asie du Sud-Est doit bien considérer les remarques faites par Grange au sujet des pays à orientation à long terme comme ceux de l'Asie du Sud-Est.
- Du point de vue de l'observateur africain, au regard des variables en présence, les distances culturelles sont aussi assez grandes. L'Africain devrait donc comprendre que les points de vue relatifs à sa sagesse traditionnelle ne sont pas toujours du goût des chinois. Car ici la différence fondamentale entre mentalités africaines et asiatiques se trouve au niveau de la dimension « Sagesse traditionnelle ».

En tout état de cause, en matière de négociation internationale, il doit avant tout être envisagé la recherche d'une solution synergique, c'est-à-dire génératrice de profits pour les parties en négociation. Et la recherche des synergies culturelles implique un certain nombre d'aspects qui exigent d'abord la compréhension des différences culturelles. En présentant les cadres de références sur lesquels reposent les cultures africaines et asiatiques (Asie du Sud-Est), on peut mieux procéder à la mise en place des synergies culturelles dans les situations où les individus évoluant dans ces deux contextes sont en relation.

\section{CONCLUSION}

Au moment où la société actuelle traverse ce qu'il est convenu d'appeler avec Zartmann (1976) « l'âge de la négociation », au moment où l'Asie fait l'objet de beaucoup de convoitise et où l'Afrique peine encore à intégrer l'économie mondiale, au moment où «l'importance des enjeux et la complexité des obstacles attachés à la conquête des « trois triangles chi-

\footnotetext{
14. «a) pratiquez la vertu et la patience; b) résignez-vous à de longue périodes d'inaction; c) gardez-vous d'entretenir des attentes exagérées, et imputez à la rhétorique chinoise les brillantes perspectives qu'on fait miroiter devant vous; d) attendez-vous à ce que, pour vous influencer, les Chinois jouent votre propre jeu; e) résistez à la tentation d'attribuer vos ennuis à vos propres erreurs; f) tout en vous efforçant de comprendre la culture chinoise, sachez qu'un étranger ne pourra jamais la vivre mieux que les Chinois eux-même. » (Pye, cité par Adler, p. 235).
} 
nois » (Chine, « Dragons » et Asie Pacifique) ont conduit un nombre croissant d'ingénieurs et de cadres commerciaux, de chercheurs et de consultants à s'initier aux arcanes de la négociation chinoise » (Pluchart, 1996); au moment où la création des zones de libre échange entre pays limitrophes renforce la régionalisation des échanges surtout dans le contexte asiatique (Ramon et al., 2003; Proff, 2002; Jain, 1999) et où le sentiment national affecte parfois de manière décisive la négociation des affaires comme c'est le cas en Chine (Shi et Wright, 2003) ; l'interrogation relative à l'influence des valeurs culturelles dans les relations d'affaires entre l'Afrique et l'Asie présente toute son importance et son actualité.

Sur la base des éléments issus de trois enquêtes cross-culturelles, il est établi que l'orientation à long terme des pays asiatiques, reflet de la pensée confucéenne, contraste fortement avec les valeurs caractéristiques de « la sagesse africaine » (consensus, hopitalité, traditions). Par conséquent, comme déjà constaté par Pluchart (1996), l'Africain doit comprendre que les pratiques chinoises en matière d'efficacité des transactions s'accommodent mal d'un schéma mécaniste de la recherche $\mathrm{du}$ consensus et s'avèrent difficilement réductibles aux modèles d'analyse occidentaux (modèles généralement qualifiés d'universels).
La négociation internationale en raison de son universalité et de sa transversalité exige le recours à un champ d'observation beaucoup plus large, à des approches méthodologiques complémentaires (triangulation et stratégie multiméthode) et à une approche multidisciplinaire. Aussi, cette étude présente-t-elle des limites. Ces limites portent notamment sur le nombre de pays africains et asiatiques concernés par les études cross-culturelles ayant servi de base de raisonnement dans ce travail, sur la méthodologie employée : nous nous sommes uniquement appuyés sur les études quantitatives. Toutefois, faut-il le souligner, notre but dans ce travail était de relever les grandes différences et les similitudes entre les cultures africaines et celles de l'Asie du Sud-Est telles que décrites dans quelques études cross-culturelles où figurent les pays africains. Les points ici présentés peuvent déjà aider l'Africain qui veut s'engager dans les relations d'affaires avec les ressortissants des pays d'Asie du Sud-Est à commencer à prendre conscience qu'il existe des différences d'ordre culturel qui peuvent influencer aussi bien le succès ou l'échec de l'affaire à conclure que le processus de négociation lui-même. Ces différences et les similitudes ici présentées peuvent ainsi servir de premiers points de repère, le négociateur africain devant chercher à mieux comprendre les cultures spécifiques des partenaires de l'Asie du Sud-Est. 


\section{Bibliographie}

Adler N. J., Comportement Organisationnel, Les Éditions Reynald Goulet, Québec, 1994. Alain H., « Les experts et la décentralisation : Effets d'illusion au Cameroun », Culture et Mondialisation : Gérer par delà les frontières, Iribarne P. (d'), Le Seuil, Paris, 1998.

Arvind V. P., Habib M. M., "The Dynamics of International Business Negociations", Business Horizons, May-June 1996.

Balandier G., Sociologie Actuelle de L'Afrique noire, 4édition, Paris, PUF, 1982.

Bollinger D., Hofstede G., Les différences culturelles dans le management, Paris, les Éditions d'Organisation, 1987.

Bourgoin H., L'Afrique malade du management, Éditions Jean Picollec, Paris, 1984.

Breuer J. T., Bartha P., « Français et Allemands écoutez vos différences », Havard-L'Expansion, printemps 1993.

Buller P. F., Kohls Kenneth S.A., "When Ethics Collide: Managing Conflicts Accross Cultures", Organizational Dynamics, vol. 28, n 4, 2000, p. 52-66.

Fouda M. O., La dimension culturelle du management dans les organisations camerounaises, Thèse de doctorat, université de Yaoundé II, avril 2003.

Fouquin M., « Le rôle des grands pays émergents dans l'industrie mondiale, 1995-2005 », Bulletin Le 4 Pages, ${ }^{\circ}$ 103, février 1999.

Grange J. M, Profession: cadre international, Tirer profit des différences culturelles dans les négociations, Les Éditions d'Organisation, Paris, 1997.

Henry A, «Vers un modèle de management africain », Cahiers d'Études Africaines, 124 XXXI-4, 1991, p. 447-473.

Hernandez E.-M., « Afrique : L'actualité du modèle paternaliste », Revue française de gestion, $\mathrm{n}^{\circ} 128$, mars-avril 2000, p. 98-106.

Hofstede G., Vivre dans un monde multiculturel, Les Éditions d'Organisation, Paris, 1994.

Hofstede G., Culture's Consequences: Comparing Values, Behaviors, Institutions and Organisation Across Nations, Sage Publications, London, 2001.

Hofstede G., Bond H. M., "Hofstede's Culture Dimensions. An Independant Validation Using Rokeach's Value Survey", Journal of Cross-Cultural Psychology, vol. 15, $\mathrm{n}^{\circ} 4$, décembre 1984, p. 417-433.

Iribarne P. (d'), «Cultures nationales et économie internationale », Futurible, février 1990, p. $45-55$.

Jain S. C., "Prospects for South Asian free trade agreement: problems and challenges", International Business Review, 8, 1999, p. 399-419.

Kamdem E., Management et interculturalité en Afrique : Expérience camerounaise, Les Presses de l'université de Laval et L'Harmattan, Paris, 2002.

Kogut B., Singh H., "The effect of national culture on the choice of entry mode", Journal of International Businness Studies, vol. 19, 1988, p. 441-432.

Laburthe-Tolra P., Les Seigneurs de la Forêt, Paris, Publications de la Sorbonne, 1981. 
Luo Y. "Time-based experience and international expansion: The case of an emerging economy”, cité par Hofstede G. dans Culture's Consequences: Comparing Values, Behaviors, Institutions and Organisation Across Nations, Sage Publications, London, 2001.

Marks M. L., Mirvis P. H., "Managing Mergers, Acquisitions, and Alliances: Creating an effective structure", Organizational Dynamics, Winters 2000.

Mveng E, Histoire du Cameroun, Tome 1, Yaoundé, CEPER, 1984.

Nancy B. J., Scott D., "Reflections on generalization of agency theory: cross-cultural considerations", Human Ressource Management Review, 14, 2004, p. 325-335.

Noorderhaven N. G., Tidjani B., "Culture, Gouvernance, and Economic Performance: An Explorative Study with a Special Focus on Africa", International Journal of Cross Cultural Management, vol. 1, 2001, p. 31-51.

Pan Y., "Influences on foreign equity ownership level in joint venture in China", cité par Hofstede G. dans Culture's Consequences: Comparing Values, Behaviors, Institutions and Organisation Across Nations, Sage Publications, London, 2001.

Peterson R. M., Dibrell C. C., Pett T. L., "Long-vs. Short term performance perspectives of Western European, Japanese, and U.S countries: Where do they lie?", Journal of World Business, 37, 2002, p. 245-255.

Phatak A. V., Habib M. M., "The Dynamics of International Business Negociations”, Business Horizons, May-June 1996.

Plantey A., La Négociation Internationale au XXIe Siècle, CNRS Éditions, Paris, 2002.

Pluchart J.-J., «La stratégie chinoise de négociation», http://www.strategie-aims.com/lille/ com5802.pdf, 1996.

Proff H., "Business unit strategies between regionalisation and globalisation", International Business Review, 11, p. 231-250

Ramon C., Edmonds C., Wallack J. S., "Asian Regionalism and its effects on trade in the 1980s and 1990s", Journal of Asian Economics, 14, 2003, p. 91-129.

Rojot J., La Négociation, Vuibert, Paris, 1994

Shi X., Wright P. C., "The potential impacts of national feelings on international business negotiations: a study in the China context", International Business Review, 12, 2003, p. 311-328.

The Chinese culture connection (a team of 24 researchers), "Chinese values and the search for culture-free dimensions of culture”, Journal of Cross-Cultural Psychology, vol. 18, 1987, p. 143-164.

Tse D. K., Pan Y., Au K. Y., "How MNC's choose entry modes and form alliances: The China experience", cité par G. Hofstede dans Culture's Consequences: Comparing Values, Behaviors, Institutions and Organisation Across Nations, Sage Publications, London, 2001.

Yadong L., "Toward a conceptual framework of international joint ventures negotiations", Journal of International Management, 5, 1999, p. 141-165.

Zartman I. William, "Fifty Per Cent Solution: How to Bargain Successfully with Hijackers, Strikers, Bosses, Oil Magnates, Arabs, Russians and Other Worthy Opponents in This Modern World ”, cité par Rojot J., La Négociation, Vuibert, Paris, 1994. 